\title{
Electron loss of fast projectiles in the collisions with molecules
}

\author{
V.I. Matveev, ${ }^{1, *}$ D.N. Makarov, ${ }^{1}$ and Kh.Yu. Rakhimov ${ }^{2}$ \\ ${ }^{1}$ Pomor State University, 4 Lomonosov St., 163002 Arkhangelsk, Russia \\ ${ }^{2}$ Department of Physics, National University of Uzbekistan, 100174 Tashkent, Uzbekistan
}

(Dated: November 13, 2018)

\begin{abstract}
The single and multiple electron loss of fast highly charged projectiles in the collisions with neutral molecules are studied within the framework of a nonperturbative approach. The cross sections for single, double, and triple electron losses are calculated for the collision system $\mathrm{Fe}^{q+} \rightarrow \mathrm{N}_{2}(q=24,25$, 26) at the collision energies 10,100 , and $1000 \mathrm{MeV} / \mathrm{u}$. The effects caused by the collision multiplicity and the orientation of the axis of target molecule are treated. It is shown that collision multiplicity effect leads to considerable differences for the cases of perpendicular and parallel orientations of the molecular axes with respect to the direction of the projectile motion, while for chaotic orientation such effect is negligible.

PACS numbers: 34.50.DB; 34.10.+x
\end{abstract}

\section{INTRODUCTION}

Stripping, or electron loss by heavy projectiles in the collisions with atoms has been subject of extensive studies during last decade. Various approach for the theoretical study of the projectile electron loss cross sections in the collisions with neutral targets are presented in the Ref. [1] and in the monograph by A. Voitkiv and J. Ullrich 2] where detailed discussion of the experimental results can be found. In theoretical study of such processes it is important the treatment of the effects caused by strong field of the projectile and accounting the transitions in the target state. Such a treatment requires using nonperturbative methods for the calculation of stripping cross sections [3, 4]. Recently such an approach was developed on the basis of sudden perturbation approximation and used for the study of electron losses by fast highly charged projectiles with neutral gas atoms [5, [6].

Despite the fact that considerable progress is made in theoretical study of electron loss by fast projectiles, most of the treatments are restricted by considering atomic targets.

However, study of the electron loss by fast highly charged projectile in the collisions with molecular targets is of fundamental and practical importance because of the number of new effects that cannot be observed in the case of atomic targets.

One of such effects is the strong dependence of the ionization (both for projectile and target) cross section, on the orientation of the molecular axis with respect to the direction of the collision velocity. Earlier the existence of such an effect was mentioned in the Refs. [7 9 ], where the ionization of molecular targets in their collisions fast highly charged ions is studied.

Another important effect that appears in the collision of fast highly charged projectiles with molecules is socalled collision multiplicity effect [10]. It implies that

*Electronic address: matveev.victor@pomorsu.ru after the collision with the first atom of target before the relaxation the projectile collides with the second atom being in the excited state. Considerable contributions by multiplicity effects to the ionization cross section and energy losses of fast highly charged ions with diatomic molecules [10] and nanoparticles [11] was found in recently.

In particular, it was shown in the Refs. [10, 11] that the orientation and multiplicity effects will occur when the time interval between two subsequent collisions is less than the relaxation time. Furthermore, it is clear that the multiple collision of the projectile with target molecule atoms occurs when the direction of the projectile motion is close to the orientation of target molecule axis. Qualitatively, the role of the orientation effects can be understood for diatomic molecule as follows. Therefore in the study of fast collisions of highly charged projectiles with diatomic molecules one should take into account the two-step processes which include collisions of the projectile with the first atom of the molecule and cannot relax into the ground state before the collision with the second atom of the target. We note that in the present paper we consider the case when the time between two subsequent collisions is much shorter than the characteristic period of the projectile electrons. Therefore the processes we deal with can be interpreted as the variation of the "atomic-double-slit" processes in which projectile electrons interacts with both molecular centers in a coherent way (see, e.g., 12]). It is important to note that the collision multiplicity effects contribute only to the projectile ionization/excitation cross section without causing any changes in the transitions of target states. Indeed, in subsequent collisions with each atom of the target molecule the ionization or excitation occurs in different atoms. It is clear that the above arguments are true for polyatomic molecules, too.

In this paper we develop a nonperturbative approach which is based on the use of sudden perturbation approximation and utilizes it for the calculations of the electron loss cross sections of fast highly charged projectiles in the collisions with polyatomic molecules. The method allows 
to take into account all the transitions both in target and projectile electronic states. Moreover, it is possible to achieve considerable simplifications of the expressions for the electron loss cross sections in the cases of high enough projectile charges and multi-electron targets .

The calculations of the electron loss cross sections and their dependence on the orientation of the molecular axis are presented for single-, double-, and triple- stripping of the fast highly charged iron ions. It is also shown that the collision multiplicity effect leads to considerable difference between the stripping cross sections for the cases of parallel and perpendicular orientation of the molecular axis with respect to the direction of projectile motion. However, for chaotic orientation of the axis of target molecule such effect is negligible. Finally, it should be noted that for the calculation of the multiple stripping cross section of the fast highly charged projectiles in their collisions with multi-electron targets one should use non-perturbative methods, since the Born approximation is not applicable for these processes. Our approach also can be considered as a nonperturbative one. However, our results concerning on the dependence of the cross sections on the molecule's axis orientation can be obtained within the perturbative approach, too: at least, for a single-electron ionization.

This paper is organized as follows. In the next section we will present detailed description of the approach to be utilized, derivation of the nonperturbative expressions for the stripping cross section and its applications for the calculations of the multiple stripping cross sections of fast highly charged projectiles in the collisions with the nitrogen molecule, $N_{2}$. The section III presents discussions of the results, while in the section IV we give some concluding remarks.

\section{THEORETICAL BACKGROUND}

The system we are going to treat includes a fast highly charged projectile colliding with a molecule that consists of multi-electron (number of electrons, $N_{A} \gg 1$ ) atoms. For such collision system the cross section for excitation or ionization of the projectile from state $|0\rangle$ to $|k\rangle$ (provided arbitrary transition can occur in the target state) with high accuracy (error is proportional to $1 / N_{A}$ ) in the Glauber approximation can be written as [6, 10]

$$
\sigma=\int\left|\left\langle k\left|\exp \left\{-\frac{i}{v} \int_{-\infty}^{+\infty} U d X\right\}\right| 0\right\rangle\right|^{2} d^{2} \mathbf{b} .
$$

Here $\mathbf{v}$ is the projectile velocity and the $x$-axis is directed along the vector $\mathbf{v}$. If $\mathbf{b}$ is the impact parameter, and target position is fixed with one of the nuclei being at the origin of coordinate system, then the position of the projectiles nucleus can be given by the vector $\mathbf{R}=(X, \mathbf{b})$. Atomic units are used here and in the following. In Eq. (1) the potential $U$ describes interaction of the projectile electrons with the target which is considered as a lengthy object. In other words, the cross section in Eq. (1) is expressed in terms of the charge density of target electrons [10, 11]). Formally, Eq. (10) has the same form as that for the ionization cross section in the "frozen" approximation for the target electrons. However, as it was shown in the Ref. [4], for the multielectron targets $\left(N_{A} \gg 1\right)$ this formula describes (with the error order of $1 / N_{A}$ ) the transition cross section for the projectile electrons summed over the complete set of final states of the target electrons. For a molecule consisting of multielectron atoms the electronic density is almost the same as the sum of that for isolated atoms. Therefore for such case we can consider the target as consisting of isolated and non-interacting atoms [10, 11]). We describe the charge density of the each atom in the target within the Hartree-Fock-Slater model [13] in which the spatial charge density can be written as

$\rho_{m}(r)=-\frac{Z_{m}}{4 \pi r} \sum_{i=1}^{3} A_{m, i} \alpha_{m, i}^{2} e^{-\alpha_{m, i} r}, \quad \sum_{i=1}^{3} A_{m, i}=1$,

where $Z_{m}$ is the charge of the $m$-th atomic nucleus, $A_{m, i}$ and $\alpha_{m, i}$ are the tabulated constants that can be found in the Ref. [13]. The potential created by molecule at the point $\mathbf{r}$ can be written in terms of potentials created by each atom of the molecule:

$$
\varphi(\mathbf{r})=\sum_{m=1}^{N} \frac{Z_{m}}{d_{m}} \Phi_{m}\left(d_{m}\right)
$$

where $N$ is the number of atoms in target, $d_{m}$ is the distance from the $m$ the nucleus in the target to the observation point, $\mathbf{r}$ and $\Phi_{m}(r)$ is the screening function for the $m$-th atom. In the Hartree-Fock-Slater model screening function can be written as:

$$
\Phi_{m}(r)=\sum_{i=1}^{3} A_{m, i} \exp \left(-\alpha_{m, i} r\right)
$$

Furthermore, let us introduce the following notations: $\mathbf{r}_{p}$ is the coordinate of the projectile electrons with respect to its nucleus, $\left(p=1,2, \ldots, N_{P}\right), N_{P}$ is the total number of projectile electrons, $\mathbf{R}_{m}=\left(X_{m}, \mathbf{b}_{m}\right)$ are the distances between the projectile nucleus and the nucleus of the $m$-th atom of the target. If $\mathbf{b}_{m}$ is the impact parameter with respect to $m$-th atom, then $d_{m}=\left|\mathbf{R}_{m}+\mathbf{r}_{p}\right|$.

Therefore the interaction potential between the target and projectile electrons can be written as

$$
U=-\sum_{p=1}^{N_{P}} \varphi\left(\mathbf{r}_{p}\right)=-\sum_{m=1}^{N} \sum_{p=1}^{N_{P}} \frac{Z_{m}}{\left|\mathbf{R}_{m}+\mathbf{r}_{p}\right|} \Phi_{m}\left(\left|\mathbf{R}_{m}+\mathbf{r}_{p}\right|\right) .
$$

Taking into account Eq. (44) for the "eikonal phase" 
involved into Eq. (1) we have

$$
\begin{gathered}
\chi=-\frac{1}{v} \int_{-\infty}^{+\infty} U d X \\
=\sum_{m=1}^{N} \frac{2 Z_{m}}{v} \sum_{p=1}^{N_{P}} \sum_{i=1}^{3} A_{m, i} K_{0}\left(\alpha_{m, i}\left|\mathbf{b}_{m}+\mathbf{s}_{p}\right|\right),
\end{gathered}
$$

with $K_{0}(z)$ being the lowest-order McDonald function and $\mathbf{s}_{p}$ is the projection of the vector $\mathbf{r}_{p}$ onto the impact parameter plane. As it was shown in the Ref. [6] Eq. (1) with the "eikonal phase" given in the formula (5) is applicable for relativistic collisions, too. Thus Eq. (1) describes the cross section for the transition of the projectile electrons from the initial state $|0\rangle$ to a state $|k\rangle$, under the assumption that arbitrary transitions can occur in the states of target electrons. The accuracy of the formula depends on the number of target electrons, $N_{A}$ i.e., the error is of order of the quantity $\sim 1 / N_{A}$ for $N_{A} \gg 1$.

In the following we will consider the highly charged projectiles whose "visible" (effective) charge, $Z_{P}$ is much larger than unity (for instance, for the $F e^{10+}$ projectile $Z_{P}=10$ while nucleus charge is $Z=26$ ). Then the characteristic size of the projectile is much less than that of an atom of the target molecule. Therefore we can assume that the mean field created by atom acts uniformly to the projectile electrons that corresponds to expansion of the "eikonal phase" in Eq. (5) in terms of a small parameter, $s_{p} / b$. This allows us to rewrite Eq. (10) for orthogonal $|0\rangle$ and $|k\rangle$ in the following form

$$
\sigma=\int\left|\left\langle k\left|\exp \left(-i \sum_{m=1}^{N} \mathbf{q}_{m} \sum_{p=1}^{N_{P}} \mathbf{r}_{p}\right)\right| 0\right\rangle\right|^{2} d^{2} \mathbf{b},
$$

where

$$
\mathbf{q}_{m}=\frac{2 Z_{m}}{v} \sum_{i=1}^{3} \alpha_{m, i} A_{m, i} K_{1}\left(\alpha_{m, i} b_{m}\right) \frac{\mathbf{b}_{m}}{b_{m}},
$$

has the meaning of the momentum transfer to each projectile electron due to the collisions with $m$-th atom of the target, $K_{1}(z)$ is the first-order McDonald function.

Furthermore, we note that Eq. (6) can be applied for the collisions of highly charged $\left(Z_{P} \gg 1\right)$ projectiles with the neutral molecules consisting of multielectron atoms. This requires fulfilling by $Z_{t}$ the condition $Z_{t} \gg 1$, with $t=1,2, \ldots, N$ being the number of atoms in target, i.e., $Z_{1}$ is the nucleus charge of the first atom of the target, $Z_{N}$ is the nucleus charge of the $N$-th atom etc.

Since the expression for the cross section is derived within the Glauber approximation, the energy of projectile, $E$ should be much larger than that of projectiletarget interaction, $U$, i.e., $E \gg U$ and $k L \gg 1$, where $k$ is the projectile momentum and $L$ is the interaction radius for the potential $U$. In the case of neutral molecular target whose size is much larger than that of projectile, as the quantity $L$ one can take characteristic size of target. It is clear that this condition is fulfilled for fast highly charged projectile. In addition, to make use our approach we have to assume that the sudden perturbation approximation is valid, i.e., for each target atom (interaction) collision time, $\tau_{c} \sim L / v$ between the projectile and target is much shorter than the period of most fastest (inner) electron, $\tau_{e}$, to be ionized:

$$
\tau_{c} \ll \tau_{e} .
$$

Fulfilling of this condition means that target electrons cannot change their position during the collision time. In this case target electrons can considered as in fixed positions during the collision [14]. For relativistic projectiles the above condition can be written as $\sqrt{1-v^{2} / c^{2}} L / v \ll \tau_{e}$. The quantity $\tau_{e}$ can be estimated for each fixed collision system. For multielectron, collision system, with most of the electrons to be ionized being in outer shells we have $\tau_{e} \sim 1$.

As it was mentioned above in order to make use Eq. (6) for the calculation of the projectile stripping cross sections in the collision with neutral molecular targets the following condition should be fulfilled

$$
Z_{P}=Z-N_{P} \gg 1,
$$

where $N_{P}$ is the number of projectile electrons before the collision, $Z$ is the charge of the projectile nucleus. This means that (unscreened) charge of projectile should be large enough to consider the projectile as highly charged particle. Important point in the calculations of projectile's multiple stripping cross section in the collisions with polyatomic molecules is the effects of collision multiplicity and effects of molecular axis orientations. To include these effects into consideration we need to assume that two or more atoms in the target are on the same line which is parallel to the vector $\mathbf{v}$. To demonstrate collision multiplicity and molecular axis orientation effects we consider simplest target, diatomic molecule. We will use notation $\mathbf{L}$ to denote the orientation vector is of the molecular axis and assume that it is directed along the line connecting nuclei of two atoms in the molecule. Then the cross section presented by formula (6) is the function of vector $\mathbf{L}$ i.e., $\sigma=\sigma(\mathbf{L})=\sigma(\theta, \phi)$. Furthermore, we represent vector $\mathbf{L}$ in terms of spherical coordinates with the angles $\phi, \theta$ and assume the molecular axis to be directed along the vector $\mathbf{v}$.

In the following we will be interested in the stripping cross section averaged over the angle $\phi$ :

$$
\sigma(\theta)=\frac{1}{2 \pi} \int \sigma(\theta, \phi) d \phi .
$$

Angle $\theta$ will be called the orientation angle of the molecular axis. Then the multiplicity effect can be characterized in terms of $\theta$-dependent relative correction, $\delta$ defined as $\delta=\left(\sigma(\theta)-\sigma_{\perp}\right) / \sigma_{\perp}$, where $\sigma_{\perp}$ is the stripping cross section at $\theta=\pi / 2$. It is easy to see that $\sigma_{\perp}$ described the collision when target moleculae axis is perpendicular to the projectile direction. 


\section{RESULTS AND DISCUSSION}

Let us now apply Eq. (6) to the collision system consisting of fast $\mathrm{Fe}^{q+}$ projectile and $\mathrm{N}_{2}$ molecule. For simplicity we consider the cases when $q=24,25,26$. Let us start from the most simplest case: stripping of hydrogenlike projectile in the collision with diatomic molecule for which the cross section can be written as

$$
\sigma(\theta, \phi)=\int d^{2} b \int d \mathbf{k}\left|\left\langle\mathbf{k}\left|\exp \left\{-i\left(\mathbf{q}_{1}+\mathbf{q}_{2}\right) \mathbf{r}\right\}\right| 0\right\rangle\right|^{2}
$$

Here $\mathbf{r}$ is the coordinate of the projectile electron with respect to the projectile nucleus, $\mathbf{k}$ is the momentum of the projectile electron lost $\mathbf{q}_{j}$ is the momentum transfer that can be written as $\mathbf{q}_{j}=\frac{2 Z_{A}}{v} \sum_{i=1}^{3} \alpha_{i} A_{i} K_{1}\left(\alpha_{i} b_{j}\right) \frac{\mathbf{b}_{j}}{b_{j}}$, where $\mathbf{b}_{j}$ is the impact parameter with respect to $j$ th nucleus of the target $(j=1,2)$.

Using Eqs. (8) and (9) we have calculated the stripping cross section $\sigma(\theta)$, of the hydrogen-like projectile $F e^{25+}$ in the collision with nitrogen molecule $N_{2}$, for different orientation angles and collision energies. A quantity we are interested to analyze is the correction to the projectile stripping cross section due to the collision multiplicity which is given as $\delta(\theta)=\left(\sigma(\theta)-\sigma_{\perp}\right) / \sigma_{\perp}$. In Fig. 1 the results of calculation of such quantity for collision system $\mathrm{Fe}^{25+}-\mathrm{N}_{2}$ are presented. Continuous line in this figure represents $\delta(\theta)$ for the collision energy $10 \mathrm{MeV} / \mathrm{u}$, while long-dashed and short-dashed lines are the results for the energies $100 \mathrm{MeV} / \mathrm{u}$ and $1000 \mathrm{MeV} / \mathrm{u}$, respectively. The angle $\theta$ is given in radians.

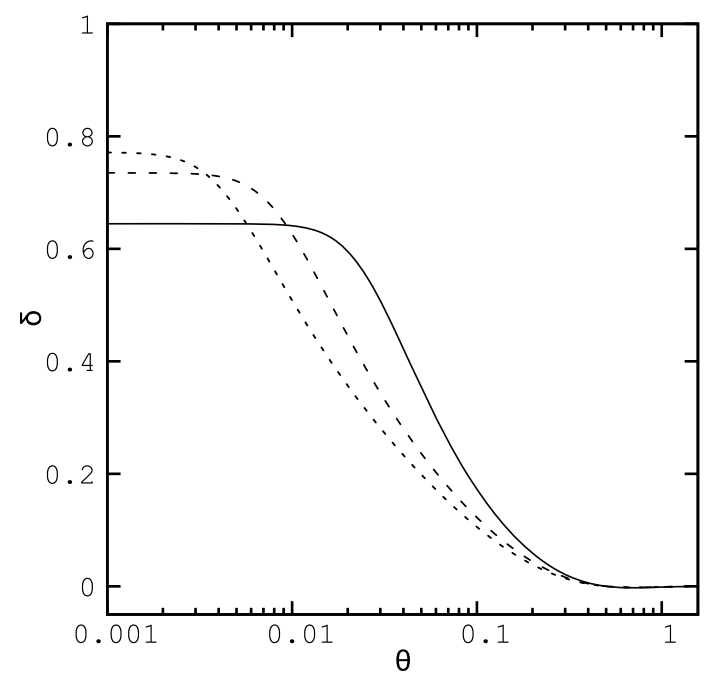

FIG. 1: The dependence of the quantity $\delta(\theta)=(\sigma(\theta)-$ $\left.\sigma_{\perp}\right) / \sigma_{\perp}$ on the orientation o target molecule axis $\theta$ for the collisions system $\mathrm{Fe}^{25+} \rightarrow N_{2}$ with $\sigma_{\perp}=\sigma(\theta=\pi / 2)$. Continuous line is the result for the collision energy $10 \mathrm{MeV} / \mathrm{u}$; longdashed line is the result for the collision energy $100 \mathrm{MeV} / \mathrm{u}$ and short-dashed line is $\delta(\theta)$ for the energy $1000 \mathrm{MeV} / \mathrm{u}$. The values of $\theta$ are given in radians.

Now let us consider stripping of helium-like projectile
$F e^{25+}-N_{2}$, in the collision with $N_{2}$ molecule. In this case according to Eq. (6) the cross section for the transition of projectile from state $|0,0\rangle$ to $\left|n_{1}, n_{2}\right\rangle$ can be written as

$$
\sigma(\theta, \phi)=\int d^{2} b\left|\left\langle n_{1}, n_{2}\left|e^{-i\left(\mathbf{q}_{1}+\mathbf{q}_{2}\right)\left(\mathbf{r}_{1}+\mathbf{r}_{2}\right)}\right| 0,0\right\rangle\right|^{2}
$$

with $\mathbf{r}_{1}$ and $\mathbf{r}_{2}$ being the coordinates of the projectile electrons with respect to projectile nucleus.

Following the Refs. [15, 16]) in our calculations (final and initial) two-electron states of the projectile are described as a symmetric products of one-electron hydrogen-like wave functions with effective charges equal to degree of ionization. Single $\sigma^{1+}(\theta)$, and double $\sigma^{2+}(\theta)$, electron loss cross sections of can be calculated using Eq. (10). Here the cross section for double stripping $\sigma^{2+}(\theta)$, corresponds to transition of both electron into the continuum state, while the single projectile ionization cross section $\sigma^{1+}(\theta)$, describes transition of one of the electrons into the continuum, by exciting another one into a state of the discrete spectrum. Correspondingly, the quantity $\delta$ can be estimated for both cases. In Figs. $2 \mathrm{a}, 2 \mathrm{~b}$, and $2 \mathrm{c}$ we plotted $\delta$ as a function of orientation $\delta$ for the collision energies 10,100 , and $1000 \mathrm{MeV} / \mathrm{u}$, respectively. Continuous line in this figure is the result for single electron loss, $\delta=\left(\sigma^{1+}(\theta)-\sigma_{\perp}^{1+}\right) / \sigma_{\perp}^{1+}$, while dashed line describes calculation of $\delta$ for double ionization, i.e., $\delta=\left(\sigma^{2+}(\theta)-\sigma_{\perp}^{2+}\right) / \sigma_{\perp}^{2+}$.

Finally, we utilized Eq. (6) for the calculation of multiple stripping of three-electron (Lithium-like) projectile, $\mathrm{Fe}^{23+}$ in the collision with $N_{2}$ molecule. In this case the loss cross section describing transition from state $|0,0,0\rangle$ to $\left|n_{1}, n_{2}, n_{3}\right\rangle$ can be written as

$$
\sigma=\int d^{2} b\left|\left\langle n_{1}, n_{2}, n_{3}\left|e^{-i\left(\mathbf{q}_{1}+\mathbf{q}_{2}\right)\left(\mathbf{r}_{1}+\mathbf{r}_{2}+\mathbf{r}_{3}\right)}\right| 0,0,0\right\rangle\right|^{2},
$$

where $\mathbf{r}_{1}, \mathbf{r}_{2}$, and $\mathbf{r}_{3}$ are coordinates of projectile electrons.

As in the case of helium-like projectile, the wave functions of three electron state are taken as the symmetric product of one-electron (hydrogen-like) wave functions with effective charges equal to degree of ionization discussed in the Refs. [15, 16]). Single, double, and triple electron loss cross sections of $\mathrm{Fe}^{23+}$ projectile can be calculated using Eq. (11). Single electron loss implies ionization of one electron, while other two electrons can be excited into any state of the discrete spectrum. Similarly, in the case if double electron loss two electrons are lost, while other electron excited into any bound state. As in the cases of single and double electron losses, we have calculated the quantity, $\delta$ for the cases of single-, double-, and triple-electron losses. Figs. 3a, 3b, and 3c present the results of calculation of $\delta(\theta)$ for the collision energies 10,100 , and $1000 \mathrm{MeV} / \mathrm{u}$, respectively. Continuous line in this figure is the result for single electron loss, $\delta=\left(\sigma^{1+}(\theta)-\sigma_{\perp}^{1+}\right) / \sigma_{\perp}^{1+}$, long dashed line describes calculation of $\delta$ for double ioniza- 

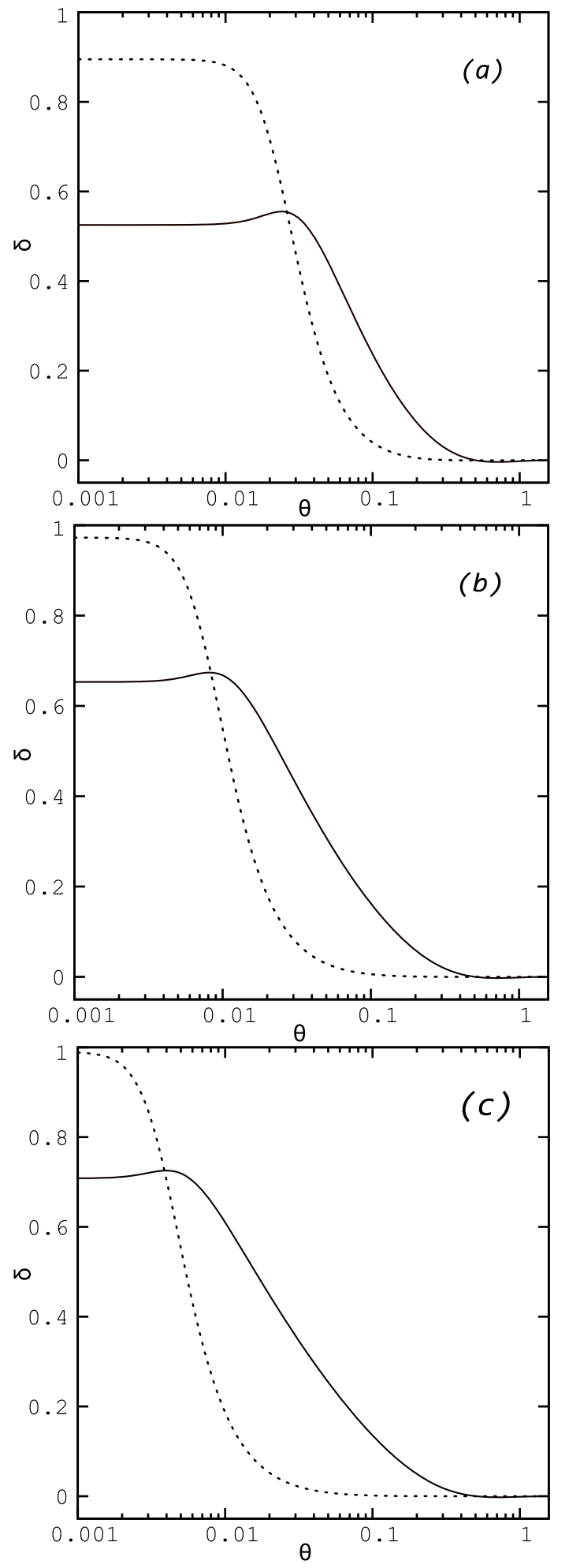

FIG. 2: The dependence of the quantity $\delta(\theta)=(\sigma(\theta)-$ $\left.\sigma_{\perp}\right) / \sigma_{\perp}$ on the orientation o target molecule axis $\theta$ for the collision system $\mathrm{Fe}^{24+} \rightarrow \mathrm{N}_{2}$ for the energies 10 (Fig. 2a), 100 (Fig. 2b), and $1000 \mathrm{MeV} / \mathrm{u}$ (Fig. 2c). Continuous line is the result for single electron loss, dashed line is the result for double electron loss by projectile. tion, $\delta=\left(\sigma^{2+}(\theta)-\sigma_{\perp}^{2+}\right) / \sigma_{\perp}^{2+}$ and short-dashed line is the plot of $\delta=\left(\sigma^{3+}(\theta)-\sigma_{\perp}^{3+}\right) / \sigma_{\perp}^{3+}$.
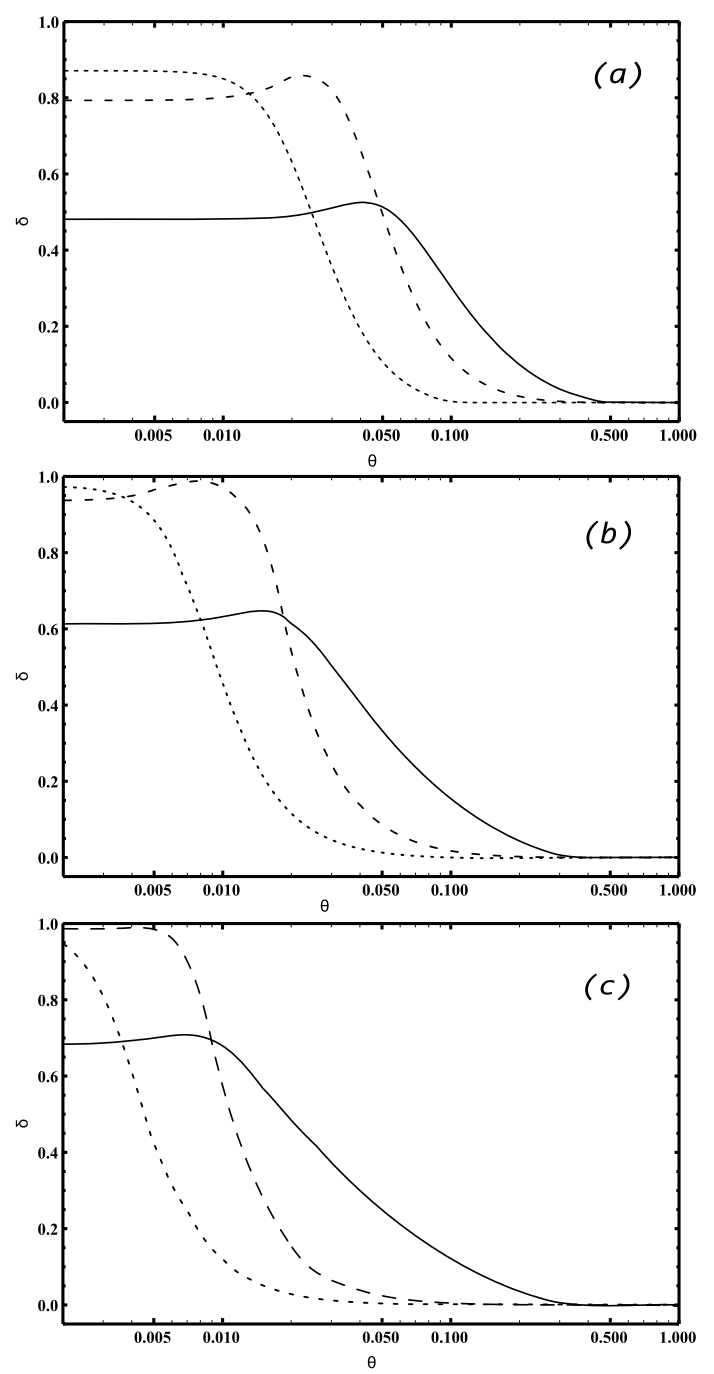

FIG. 3: The dependence of the quantity $\delta(\theta)=(\sigma(\theta)-$ $\left.\sigma_{\perp}\right) / \sigma_{\perp}$ on the orientation o target molecule axis $\theta$ for the collision system $\mathrm{Fe}^{23+} \rightarrow N_{2}$ for the energies 10 (Fig. 3a), 100 (Fig. 3b), and $1000 \mathrm{MeV} / \mathrm{u}$ (Fig. 3c). Continuous line is the result for single electron loss, long-dashed line is the result for double electron loss and short-dashed line for triple electron loss by projectile.

As is seen from the Figs. 1-3, for all collision energies stripping cross sections considerably depend on the orientation angle $\theta$, the difference between "perpendicular" and "parallel" orientations is about $50 \%-100 \%$. It is easy to estimate from the geometrical analysis the value of the angle $\theta$ at which multiplicity effect becomes essential. Since in all cases projectile collides with the neutral atoms of the target molecule whose sizes $\sim 1$, for the internuclear distance of target atoms denoted by $L$, the orientation can be estimated as $\theta \leq 1 / L$. Therefore for the nitrogen molecule, for instance, we have $L=2.07$, that gives the estimate $\theta \leq 0.5$. This can be seen also from the Figs. 1-3. 
Also, it should be noticed that the behavior of the quantity $\delta$ almost the same for all collisions partners. To check this we have done (similar to the above) calculations for one-, two-, and three-electron $\mathrm{Ni}$ and $\mathrm{Xe}$ projectiles colliding with $\mathrm{N}_{2}, \mathrm{O}_{2}$, and $A u_{2}$ targets. The difference from the above treated collision systems we observed for these systems was too small to present them in this paper.

Finally, since in the experiment the cross section for chaotic orientation are usually measured, we have done calculations of the stripping cross section averaged over the angle $\theta$ assuming uniform distribution on the orientation angle:

$$
\bar{\sigma}=\int \sigma(\mathbf{L}) \frac{d \Omega}{4 \pi}=\int_{0}^{\pi} \sigma(\theta) \frac{1}{2} \sin \theta d \theta .
$$

As showed the results of such calculations the corrections due to the collision multiplicity for the case of chaotic orientation is too small: order of 0.1 percents. In this case the difference between the cross sections $\bar{\sigma}$ and $\sigma_{\perp}$ is very small. Thus the collision multiplicity effect is considerable only for the case of regular (non-chaotic) orientation of the molecular axis, while for chaotic orientation it becomes negligible.

\section{CONCLUSIONS}

The electron losses of fast highly charged projectiles in the collisions with neutral molecules has been studied.
Based on the Glauber approximation a nonperturbative approach is developed to estimate single and multiple stripping cross sections. Using the method single, double, and triple electron loss cross sections of the fast $\mathrm{Fe}^{25+}$, $\mathrm{Fe}^{24+}$, and $\mathrm{Fe}^{23+}$ ions in the collisions with $\mathrm{N}_{2}$ molecule are calculated. The effect of collision multiplicity, caused by the collisions of the projectile with separate atoms of the target is analyzed. It is shown that multiplicity effect is essential for the case when the orientation of the target molecule axis is parallel or perpendicular to the projectile velocity direction, while for chaotic orientation such effect is negligible. We note that in all the cases we calculate the total electron loss cross sections, i.e., we perform summation (integration) over the all continuum states of the electron after the ionization. Therefore, in the present paper we didn't discuss the energies and directions of the emitted electrons. The above developed method is rather simple for application and can be used for any (polyatomic) target molecules and for the projectiles of arbitrary high (including relativistic) velocities.

\section{ACKNOWLEDGEMENT}

We are grateful to Davron Matrasulov for valuable discussions and useful comments. This work is supported by the grant of Russian Foundation of Basic Studies (Ref. Nr. 08-02-00711-a) and by the grant of the Russian Federation President (Ref. Nr. MK-3592.2011.2).
[1] A.B. Voitkiv, Phys. Rep. 392, 191 (2004).

[2] A.B. Voitkiv and J. Ullrich, Relativistic Collisions of Structured Atomic Particles, Vol. 49: Springer Series on Atomic, Optical, and Plasma Physics (Springer Verlag, Berlin, Heidelberg, 2008) 285 p.

[3] D. DuBois, A.C.F. Santos, Th. Stöhlker, et al. Phys. Rev. A 70, 032712 (2004).

[4] R.E. Olson, R.L. Watson, V. Horvat, et al. J. Phys. B 37, 4539 (2004).

[5] V.I. Matveev, D.U. Matrasulov, and S.V. Ryabchenko, J. Exp. Theor. Phys. 102, 1 (2006).

[6] V.I. Matveev and D.B. Sidorov, J. Exp. Theor. Phys. 105, 502 (2007).

[7] B. Siegmann, U. Werner, R. Mann, et al. Phys. Rev. A 65, 010704 (2001).

[8] Z. Kaliman, N. Orli, N.M. Kabachnik and H.O. Lutz, Phys. Rev. A 65, 012708 (2001).

[9] B. Siegmann, U. Werner, Z. Kaliman, et al. Phys. Rev.
A 66, 052701 (2002).

[10] V.I. Matveev, E.S. Gusarevich, S.V. Ryabchenko, and D.N. Makarov, JETP Lett. 88, 232 (2008).

[11] V.I. Matveev, E.S. Gusarevich, and D.N. Makarov, J. Exp. Theor. Phys. 109, 726 (2009).

[12] A.B. Voitkiv, B. Najjari, D. Fischer, et al. arXiv.org: 1103.0670v1 (2011).

[13] F. Salvat, J.D. Martinez, R. Mayol, and J. Parellada, Phys. Rev. A 36, 467 (1987).

[14] L.D. Landau and E.M. Lifshitz, Course of Theoretical Physics, Vol. 3: Quantum Mechanics: Non-Relativistic Theory (Nauka, Moscow, 1989, 4th ed.; Pergamon, New York, 1977, 3rd ed.).

[15] V.I. Matveev, S.V. Ryabchenko, D.U. Matrasulov, et al. Phys. Rev. A 79, 042710 (2009).

[16] V.I. Matveev and Kh.Yu. Rakhimov, J. Exp. Theor. Phys. 87, 891 (1998). 